\title{
Investigation of construct validity of state anxiety inventory among Iranian 8th grade students
}

\begin{abstract}
This study is aimed to investigate construct validity of State Anxiety Inventory (SAI Y-1) among 8th grade Iranian students. Data from 680 lower secondary school students, (317 male and 363 female, in the age 14 years old) at Tehran and Shahriar City, the province of Tehran, Iran, who participated in the study was analysed. Principal component analysis was performed to determine underlying constructs among items on the S-Anxiety Inventory (SAI Y-1). The results of the study showed that three factors explained internal relations between the items. Safety factor states $21.87 \%$ with 7 items, tension factor states $14.5 \%$ with 4 items and confusion factor states $12.37 \%$ with 3 items.
\end{abstract}

Keyword: State anxiety inventory; Construct validity; Iranian students 